\title{
Evaluating the expression level of HERV-K env, np9, rec and gag in breast tissue
}

\author{
Shaian Tavakolian, Hossein Goudarzi and Ebrahim Faghihloo*i(
}

\begin{abstract}
Objective: Breast cancer is one of the most common health problems. It has been suggested that several risk factors, either considered as external or internal, play a critical role in the pathogenesis of breast cancer, which among them, HERV-k, has the most fundamental role. In the present study, we aimed to evaluate the role of HERVk env, gag, rec, np9 expressions in breast cancer progression.

Materials and methods: We collected 40 breast cancer tissues and their normal adjacent ones. After extracting the RNA of breast samples, we evaluated the expression of HERV-k env, gag, rec, np9 by using Quantitative real-time PCR (qRT-PCR).

Results: The resulting data revealed that while there was a meaningful increase in the expression level of HERV-k env, gag and $n p 9$ in breast cancer tissues ( $P \leq 0.01,0.05,0.05$, respectively), we failed to find any significant elevation in the expression level of rec mRNA level.

Conclusion: The results of our study suggested that there is a plausible correlation between the mRNA expression level of HERV-K env, gag and $n p 9$ and the progression of breast cancer, proposing these markers as promising biomarkers to diagnose breast cancer.
\end{abstract}

Keywords: Env, np9, Rec, Gag, Breast cancer

\section{Introduction}

The diagnostic approaches in breast cancer, as the second most prevalent cause of cancer death in womankind $[1,2]$, still remain challenging for clinician, opening the way for the recognition of new biomarkers for early detection of this malignancy. The heterogeneous characteristic, complex etiology, diverse genetic mutation and the various distinct clinical manifestations suggested that a wide variety of internal and external risk factors could be involved in the pathogenesis of breast cancer. Apart from external risk factors, such as obesity, smoking, consumption of alcohol and the level of melatonin hormone, internal risk factors, including genetic and epigenetic could initiate signaling pathways in breast cancer through regulation of different genes [3-9].

Previous studies have declared that Human endogenous retrovirus (HERV) is one of the demanding factors in cancer progression, since this virus could stimulate

\footnotetext{
* Correspondence: faghihloo@gmail.com

Department of Microbiology, School of Medicine, Shahid Beheshti University of Medical Sciences, Tehran, Iran
}

neoplastic cells to proliferate continuously and to evade from apoptosis [10]. Upon 30-40 million years ago, since the first time that HERV has entered into the germ line cells, the genes of this virus has transmitted vertically into the host's genome [11], comprised approximately $8 \%$ of human genes. According to exogenous division, instead of phylogenetic one, HERV is classified into three classes, including class I (gammaretrovirusand epsilonretrovirus-like), class II (betaretrovirus-like) and class III (spumaretrovirus-like) [12-15]. Among all HERV families, HERV-K has integrated into cell genome more recently. Given to its set of intact open reading frames and biological activity, this sub-family of HERV could synthetize some retrovirus-like particles [16]. Moreover, it has been reported that the long terminal repeats (LTR) sequence in HERV regulates the expression of structural genes, including HERV-K $n p 9$, rec, env and gag genes [17], which could initiate autoimmune diseases and different types of cancers [18]. As the relationship between the expression of HERV-K $n p 9$, rec, env and gag and breast cancer remains to be an open to 
debate, in this study, we evaluated the expressions of HERV-K $n p 9$, rec, env and $g a g$ genes in patients suffering from breast cancer.

\section{Methods \\ Samples}

This study was approved by the Shahid Beheshti University of Medical Sciences" IR.SBMU.MSP.REC.1398.563 (Grant no 17631). For evaluating the expression level of HERV-k env, gag, rec, $n p 9$, 40 breast cancer (76.4, mean age) and their adjacent normal breast tissues, which was at least $5 \mathrm{~cm}$ away from tumor, have been collected from Emam hossein and Taleghany hospital between 2018 and 2019 in Tehran, Iran. All tissues were stored in RNA latter (Qiagen $\mathrm{GmbH}$, Hilden,Germany) in $-20^{\circ} \mathrm{C}$. The stage of malignant tissues was confirmed by expert pathologists and the information of patients is summarized in Table 1. The samples of those patients who were treated either by chemotherapy or radiation were excluded from this study.

\section{RNA extraction}

All tissues were suspended in $1 \mathrm{ml}$ RNX-plus solution reagent (Cinnagen, Tehran, Iran) in homogenizer. After adding chloroform and centrifuging, protein was extracted from solution. Afterwards, we added isopropanol to the solution to precipitate the RNA of supernatant. Finally, RNA plate was diluted with 50 ul of DEPCtreated water. To confirm the purity of RNA, all of RNA samples were run in agarose gel electrophoresis in order to observe $5 \mathrm{~S}, 18 \mathrm{~S}$ and $28 \mathrm{~S}$ bands. (To prevent the role of DNA, we used DNAse.)

\section{cDNA synthesis}

The reverse transcription reaction was performed using Bio fact cDNA kit (Daejeon, South Korea). To convert RNA into DNA, we provided a $20 \mu$ reaction containing $1 \mathrm{ul}$ random hexamer, $9 \mathrm{ul}$ master mixes and $10 \mathrm{ul}$ of RNA samples. The incubation was carried out for 40 min at $50{ }^{\circ} \mathrm{C}$ and $10 \mathrm{~min}$ at $95{ }^{\circ} \mathrm{C}$ in Bio Intellectica PCR. The synthesized cDNAs were then diluted twofold in sterile water.

Table 1 The Clinical characteristics of 40 breast cancer patients

\begin{tabular}{ll}
\hline$>60$ & $69 \%$ \\
\hline Tumor localization & (Left: 45\%) - (right: 55\%) \\
Family history & (Absent: 88.1\%) - (Present: 11.9\%) \\
Lymph node metastasis & (Negative: 48\%) - (positive: 52\%) \\
Tumor size & $(>2 \mathrm{~cm}: 62.8 \%)-(<2 \mathrm{~cm}: 37.2 \%)$ \\
Tumor stage & $($ I-II: 48\%) - (III-IV: 52\%) \\
Estrogen receptor (ER) status & (Negative: 54.3\%) - (positive: 45.7\%) \\
Progesterone receptor (PR) status & (Negative: 46.6\%) - (positive: 53.4\%) \\
\hline
\end{tabular}

\section{Quantitative real-time PCR}

Alterations in the expression level of mRNAs were assessed using Real-time RCR analysis. We have combined $10 \mathrm{ul}$ BIOFACT $^{\mathrm{Tm}} 2 \mathrm{X}$ real-time PCR master mix (for SYBR Green I; BIOFACT, South Korea), 6 ul sterile water, $1 \mathrm{ul}$ forward $10 \mathrm{pmol}, 1 \mathrm{ul}$ reverse primer $10 \mathrm{pmol}$, 2 ul cDNA in a final 20 ul volume, and incubated in one cycle at $95^{\circ} \mathrm{C}$ for $10 \mathrm{~min}, 40$ cycles at $95^{\circ} \mathrm{C}$ for $30 \mathrm{~s} ; 55^{\circ} \mathrm{C}$ for $30 \mathrm{~s}, 72^{\circ \mathrm{C}}$ for 30s in Rotor-gene 6000 (Corbett life sciences, Sydney, Australia) in 36-well Gene Discs. The melt curve was between $60^{\circ} \mathrm{C}$ and $95{ }^{\circ} \mathrm{C}$. GAPDH housekeeping gene was amplified as an internal control, and the values for the relative quantification were calculated based on $2^{-\Delta \Delta c t}$ expression formula. (For every gene, we used a negative control to demonstrate that there was no cross contamination between samples, in real-time PCR.)The list of primers used in this study is summarized in Table 2. (Alignment with BioEdit software was performed to avoid cross reaction of our primers used in this study with complete MMTV genome.)

\section{Statistical analysis}

To analyze the results of mRNA expression, we used Graph-PadPrism software. Experimental data are expressed by mean \pm standard deviation of three independent assays. Statistical significance was calculated using ANOVA tests. $P$-value less than $(P<0.05)$ was used for the differences.

\section{Results \\ Samples}

To evaluate the expression level of different HERV-K associated genes in mammary carcinoma, 40 breast cancer tissues and their normal adjacent parts were collected from Emam hossein and Taleghany hospital. All tested tissues were analyzed by two expert pathologists and it has been confirmed that more than $50 \%$ of patients possessed a tumor with stage of III-IV (Table 1).

\section{Evaluation in the expression level of HERV-K associated genes in breast cancer tissue}

To assess whether there was a correlation between the expression level of LTR-regulated genes and the progression of breast cancer, we extracted RNA from the tissues. RQPCR analysis revealed that there was a significant elevation in the mRNA level of env in comparison with the normal tissue (** $P$ value $<0.01$ ) (Fig. 1. A).

Moreover, our results showed that while the ranscription level of $n p 9$ and gag increased in breast cancer tissue, this expression remained unchanged in normal adjacent tissues, highlighting that probably there is an association between the expression of these genes and the progression of breast cancer (" $P$ value $<$ 0.05) (Fig. 1. B, C). 
Table 2 Nucleotide sequences of primers used for real-time RT-PCR

\begin{tabular}{lll}
\hline Gene & Forward primer $\left(5^{\prime}-3^{\prime}\right)$ & Reverse primer (5'-3') \\
\hline GAPDH & ATGTTCGTCATGGGTGGAA & GGTGCTAAGCAGTTGGTGGT \\
rec & ATCGAGCACCGTTGACTCACAAGA & GGTACACCTGCAGACACCATTGAT \\
np9 & AGATGTCTGCAGGTGTACCCA & CTCTTGCTTTCCCCACATTTC \\
env & TAACCCTGTCACTTGGATT & ATGTCACTGTCTCTTCGG \\
\hline
\end{tabular}

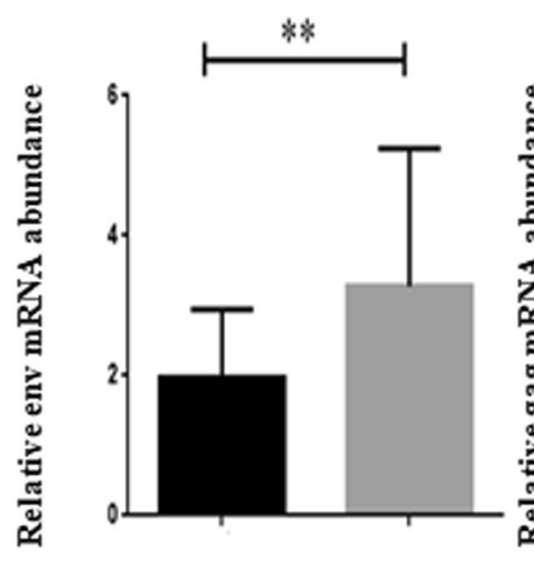

(A)
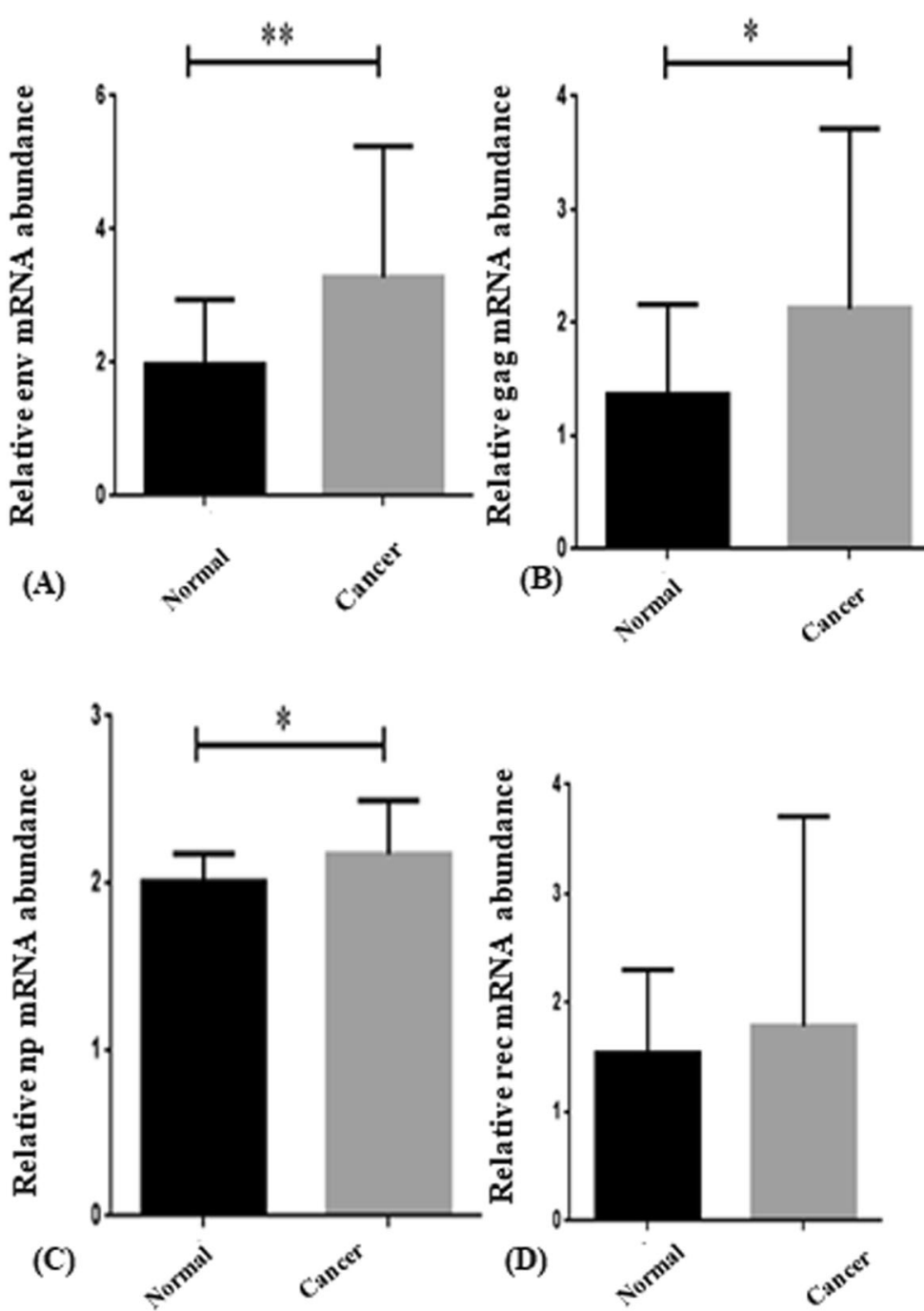

Fig. 1 a. env mRNA expression in the breast cancer compared to normal tissues. The expression level of env remarkably increased in the breast cancer tissues as compared to normal cells. ${ }^{*} P \leq 0.01$ represents significant changes from normal tissue. b. gag mRNA expression in the breast cancer tissues compared to normal tissues. The expression level of gag increased significantly in breast cancer tissue. ${ }^{*} P \leq 0.05$ represents significant changes from untreated control. c. Evaluating the mRNA expression level of np9 in normal and breast cancer tissue. The results of RQPCR analysis revealed that there is a significant elevation in the expression of np9 in breast cancer tissues as compared to normal counterparts. * $P \leq 0.05$ represents significant changes from normal tissue. (Values are given as mean \pm standard deviation of three independent experiments). $\mathbf{d}$ The comparison between the mRNA expression level of rec in the normal and breast cancer tissues 
Unlike the expression of aforementioned genes, we could not find any significant alteration in the expression level of rec either in breast cancer tissue, or in normal adjacent parts (Fig. 1. D).

\section{Discussion}

Mounting body of evidence has enumerated human endogenous retrovirus (HERV) families can be considered as one of the most important risk factors in the progression of human cancers, especially in breast cancer $[10,19]$. Among different sub-groups of HERV, the results of some studies has declared that Herv-k env, gag, $r e c$, and $n p 9$ genes could stimulate the invasive properties of cancer cells, giving them an opportunity to expand to other tissues and areas. Furthermore, the mouse mammary tumor virus (MMTV) as another risk factor, which is belonged to genus Betaretrovirus in cancer progression, cannot be ignored. However, the exact role of these viruses in the progression of breast cancer remains to be an open to debate. In the present study, we aimed to evaluate whether there was a correlation between the expression of HERV-K env, gag, rec, $n p 9$ and the progression of breast cancer.

The association between the expression of env gene and the progression of breast cancer has emerged from the recent disclosure indicating that HERV-K and MMTV env gene could initiate the progression of breast tumor through modulation of Ras/Raf/MEK/ ERK signaling pathway [20]. The results of the present study showed that env gene is over-expressed in breast cancer samples in comparison with the normal tissues. This finding was consistent with the study conducted by Johanning, et al. who showed that the expression of env increased not only in various malignant breast cell line (MDA-MB-231, MCF-7, SKBR3, MDA-MB-453, T47D, and ZR-75-1) compare to non-malignant ones (MCF-10A and MCF-10AT) [21], but also elevated during breast cancer [22]. The correlation between env and breast cancer has also been reported in several other studies [23, 24]. Moreover, in another study, it has been reported that the expression of env gene increased in invasive ductal carcinoma as compare to normal ones. Zhao et al. also delineated that both Chinese and American patients who had breast cancer displayed an over-expressed env; however, the expression of this gene was normal in healthy women [25]. Naccarato AG and et al. shown, exogenous MMTV env-like sequences (MMTVels) was presented higher in sporadic breast cancer in comparison with hereditary breast cancer; therefore this virus can be related with sporadic breast cancer [26]. Nevertheless, in some countries, such as Saudi Arabia, the sequences of MMTVels was reported low frequency [27]. Recently, Salmons B and et al. wrote a review article in relationship between MMTV and human breast cancer, and also studied on different MMTV mechanisms of oncogenesis, and they suggested further studies may confirm the effect of this virus on breast cancer invasion [28].

Another HERV-associated gene whose its expression was elevated in malignant tissues was HERV-k $n p 9$. There are several reports claiming that the expression level of this gene is increased in a variety of human cancers, ranging from solid tumors, such as breast cancer [25, 29] and melanoma [30], to chronic hematologic malignancies [31]. The oncogenic property of $n p 9$ is also evident in transformed cell lines, where it has been reported that this gene could serve as a growth factor for cancer cells [32]. The association between $n p 9$ gene and several signaling pathways, including WNT, ERK, Akt and Notch1 suggested that probably the over-expression of this gene could play a crucial role in the progression of breast cancer [33, 34]. Apart from $n p 9$, we also found that there is a significant over-expression in the mRNA level of gag. Interestingly, the relationship between gag expression and the incidence of human cancers has been evaluated in different recent studies; however, in many cases there are conflicting results. While a previous report conducted by Gary L. Johanning could not find any changes in the expression level of gag between breast cancer tissues and normal counterparts [22], other study reported that the patients with breast cancer possessed the higher mRNA level of gag [35]. The association between gag and human cancer is not restricted in breast cancer and the expression of this gene is considered as a biomarker for prostate cancer [36]. The other gene which its expression is regulated by LTR is HERV-k rec. Although the inhibitory effect of this gene on apoptosis has been reported in several human cancers, including skin [37] and ovarian cancer [38], we failed to find any association between this gene and the incidence of breast cancer, which may be due to high variation of expression levels in cancer tissues; moreover, mismatches may have an effect on the expression of rec. Klein D indicated minor mismatch, including point mutations in the primer or the probe region can lead to decrease in the quantification; conversely, major mismatches of three or four nucleotides can inhibit the real-time PCR detection, completely [39].

\section{Conclusion}

The results of the current study suggested that the expression level of HERV-K env, gag and $n p 9$ was elevated in breast cancer cells; introducing these genes as a promising biomarkers for the early diagnosis of breast cancer. 


\section{Acknowledgements}

Not applicable.

\section{Open access}

This article is distributed under the terms of the Creative Commons Attribution 4.0 International License (http://creativecommons.org/licenses/ by/4.0/), which permits unrestricted use, distribution, and reproduction in any medium, provided you give appropriate credit to the original author(s) and the source, provide a link to the Creative Commons license, and indicate if changes were made. The Creative Commons Public Domain Dedication waiver (http://creativecommons.org/publicdomain/zero/1.0/) applies to the data made available in this article, unless otherwise stated.

\section{Authors' contributions}

E.F. and SH. T designed the study and performed the molecular experiments. H. G performed the statistical Analyses. All authors read and approved the final version of the manuscript.

\section{Funding}

The present study is financially supported by the Research Department of the School of Medicine Shahid Beheshti University of Medical Sciences; IR.SBMU.MSP.REC.1398.563 (Grant no 17631).

\section{Availability of data and materials}

Please contact author for data requests.

\section{Ethics approval and consent to participate}

This study has been conducted in Department of the School of Medicine Shahid Beheshti University of Medical.s

\section{Consent for publication}

The consent for publications was obtained from all patients.

\section{Competing interests}

The authors declare no conflicts of interests.

\section{Received: 25 July 2019 Accepted: 18 November 2019}

\section{Published online: 29 November 2019}

\section{References}

1. DeSantis CE, et al. Breast cancer statistics, 2015: convergence of incidence rates between black and white women. CA Cancer J Clin. 2016;66(1):31-42.

2. Baade P. Geographical Variation in Breast Cancer Outcomes. 2017. Multidisciplinary Digital Publishing Institute.

3. Dyrstad SW, et al. Breast cancer risk associated with benign breast disease: systematic review and meta-analysis. Breast Cancer Res Treat. 2015;149(3):569-75.

4. Brouckaert $\mathrm{O}$, et al. Reproductive profiles and risk of breast cancer subtypes: a multi-center case-only study. Breast Cancer Res. 2017:19(1):119.

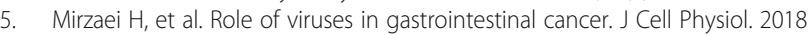
May:233(5):4000-14.

6. Faghihloo E, et al. Genotype circulation pattern of human respiratory syncytial virus in Iran. Infect Genet Evol. 2014 Mar;22:130-3.

7. Sun YS, et al. Risk factors and preventions of breast Cancer. Int J Biol Sci. 2017 Nov 1;13(11):1387-97.

8. Faghihloo $\mathrm{E}$, et al. Prevalence and characteristics of Epstein-Barr virusassociated gastric cancer in Iran. Arch Iran Med. 2014 Nov;17(11):767-70

9. Vaezjalali $\mathrm{M}$, et al. Hepatitis $\mathrm{B}$ viral DNA among $\mathrm{HBs}$ antigen negative healthy blood donors. Hepat Mon. 2013 Mar 17;13(3):e6590.

10. Gonzalez-Cao M, et al. Human endogenous retroviruses and cancer. Cancer biology \& medicine. 2016;13(4):483.

11. Boeke, J. and J. Stoye, Retrotransposons, endogenous retroviruses, and the evolution of retroelements. 1997.

12. Vargiu $L$, et al. Classification and characterization of human endogenous retroviruses; mosaic forms are common. Retrovirology. 2016;13(1):7.

13. Blomberg J, et al. Classification and nomenclature of endogenous retroviral sequences (ERVs): problems and recommendations. Gene. 2009;448(2):115-23.

14. Jern P, Sperber GO, Blomberg J. Use of endogenous retroviral sequences (ERVs) and structural markers for retroviral phylogenetic inference and taxonomy. Retrovirology. 2005;2(1):50.

15. Turner $\mathrm{G}$, et al. Insertional polymorphisms of full-length endogenous retroviruses in humans. Curr Biol. 2001;11(19):1531-5.
16. Towler EM, et al. Functional characterization of the protease of human endogenous retrovirus, K10: can it complement HIV-1 protease? Biochemistry. 1998;37(49):17137-44.

17. Dewannieux M, Blaise $\mathrm{S}$, Heidmann T. Identification of a functional envelope protein from the HERV-K family of human endogenous retroviruses. J Virol. 2005;79(24):15573-7.

18. Tugnet $\mathrm{N}$, et al. Human endogenous retroviruses (HERVs) and autoimmune rheumatic disease: is there a link? The open rheumatology journal. 2013;7:13.

19. Faghihloo E, et al. Cdc6 expression is induced by HPV16 E6 and E7 oncogenes and represses E-cadherin expression. Cancer Gene Ther. 2016 Nov; 11.

20. Zhou F, et al. Activation of HERV-K Env protein is essential for tumorigenesis and metastasis of breast cancer cells. Oncotarget. 2016;7(51):84093.

21. Wang-Johanning F, et al. Immunotherapeutic potential of anti-human endogenous retrovirus- $K$ envelope protein antibodies in targeting breast tumors. J Natl Cancer Inst. 2012;104(3):189-210.

22. Johanning $\mathrm{GL}$, et al. Expression of human endogenous retrovirus- $\mathrm{K}$ is strongly associated with the basal-like breast cancer phenotype. Sci Rep. 2017;7:41960.

23. Wang-Johanning F, et al. Human endogenous retrovirus $\mathrm{K}$ triggers an antigen-specific immune response in breast cancer patients. Cancer Res. 2008;68(14):5869-77.

24. Zhou F, et al. Chimeric antigen receptor T cells targeting HERV-K inhibit breast cancer and its metastasis through downregulation of Ras. Oncoimmunology. 2015:4(11):e1047582.

25. Zhao J, et al. Expression of human endogenous retrovirus type $\mathrm{K}$ envelope protein is a novel candidate prognostic marker for human breast cancer. Genes \& cancer. 2011;2(9):914-22.

26. Naccarato AG, et al. Mouse mammary tumor virus (MMTV) - like exogenous sequences are associated with sporadic but not hereditary human breast carcinoma. Aging (Albany NY). 2019 Sep 13;11(17):7236-41.

27. Dossary R, et al. Prevalence of mouse mammary tumor virus (MMTV)-like sequences in human breast cancer tissues and adjacent normal breast tissues in Saudi Arabia. BMC Cancer. 2018:18:170.

28. Salmons $B$, et al. Revisiting a role for a mammary tumor retrovirus in human breast cancer. Int J Cancer. 2013 Oct 1;133(7):1530-5.

29. Tavakolian S, et al. Transcriptional regulation of epithelial to Mesenchymal transition related genes by lipopolysaccharide in human cervical Cancer cell line HeLa. Asian Pac J Cancer Prev. 2019 Aug 1;20(8):2455-61.

30. Büscher $\mathrm{K}$, et al. Expression of the human endogenous retrovirus-K transmembrane envelope, rec and Np9 proteins in melanomas and melanoma cell lines. Melanoma Res. 2006;16(3):223-34.

31. Fischer $\mathrm{S}$, et al. Human endogenous retrovirus np9 gene is over expressed in chronic lymphocytic leukemia patients. Leukemia research reports. 2014;3(2):70-2

32. Armbruester $V$, et al. A novel gene from the human endogenous retrovirus K expressed in transformed cells. Clin Cancer Res. 2002 Jun;8(6):1800-7.

33. Chen T, et al. The viral oncogene Np9 acts as a critical molecular switch for co-activating $\beta$-catenin, ERK, Akt and Notch1 and promoting the growth of human leukemia stem/progenitor cells. Leukemia. 2013;27(7):1469.

34. Armbruester $\mathrm{V}$, et al. Np9 protein of human endogenous retrovirus $\mathrm{K}$ interacts with ligand of numb protein X. J Virol. 2004;78(19):10310-9.

35. Wang-Johanning $F$, et al. Human endogenous retrovirus type $\mathrm{K}$ antibodies and mRNA as serum biomarkers of early-stage breast cancer. Int J Cancer. 2014:134(3):587-95.

36. Rastogi A, et al. Autoantibodies against oncogenic ERG protein in prostate cancer: potential use in diagnosis and prognosis in a panel with C-MYC. AMACR and HERV-K Gag. Genes Cancer. 2016 Nov;7(11-12):394-413.

37. Schiavetti $F$, et al. A human endogenous retroviral sequence encoding an antigen recognized on melanoma by cytolytic T lymphocytes. Cancer Res. 2002 Oct 1:62(19):5510-6.

38. Wang-Johanning F, et al. Expression of multiple human endogenous retrovirus surface envelope proteins in ovarian cancer. Int J Cancer. 2007; 120(1):81-90.

39. Klein D. Proviral load determination of different feline immunodeficiency virus isolates using real-time polymerase chain reaction: influence of mismatches on quantification. Electrophoresis. 1999 Feb;20(2):291-9.

\section{Publisher's Note}

Springer Nature remains neutral with regard to jurisdictional claims in published maps and institutional affiliations. 